Rabaska

Revue d'ethnologie de l'Amérique française

\title{
Maheu, Ange-Émile. Mon oncle Émile conte. Sudbury, Éditions Prise de parole, 2005, 282 p. + DC. ISBN 2-89423-173-3.
}

\section{Bertrand Bergeron}

Volume 5, 2007

URI : https://id.erudit.org/iderudit/019055ar

DOI : https://doi.org/10.7202/019055ar

Aller au sommaire du numéro

Éditeur(s)

Société québécoise d'ethnologie

ISSN

1703-7433 (imprimé)

1916-7350 (numérique)

Découvrir la revue

Citer ce compte rendu

Bergeron, B. (2007). Compte rendu de [Maheu, Ange-Émile. Mon oncle Émile

conte. Sudbury, Éditions Prise de parole, 2005, 282 p. + DC. ISBN 2-89423-173-3.]

Rabaska, 5, 178-181. https://doi.org/10.7202/019055ar d'utilisation que vous pouvez consulter en ligne.

https://apropos.erudit.org/fr/usagers/politique-dutilisation/ 
À son retour du Klondike, il entreprenait en 1903 une carrière de comptable qu'il exerça jusqu'en 1914 pour différentes entreprises, avant de devenir un des assistants d'Édouard-Zotique Massicotte aux Archives judiciaires de Montréal. Il cumulera aussi diverses fonctions comme celles de maire de Saint-Constant, préfet de comté de La Prairie, agent fédéral auprès des Amérindiens de Caughnawaga, secrétaire de la Commission scolaire et agent de la Banque canadienne nationale.

Cette publication comble un vide, car il existe peu de récits relatant « l'épopée des chercheurs d'or canadiens-français ». Les lecteurs seront choyés d'en apprendre autant sur le Klondike.

Jeanne Pomerleau

Québec

Maheu, Ange-Émile. Mon oncle Émile conte. Sudbury, Éditions Prise de parole, 2005, 282 p. + DC. ISBN 2-89423-173-3.

Le recueil d'Ange-Émile Maheu, Mon oncle Émile conte, appelle un double commentaire selon qu'on l'aborde du point de vue des genres narratifs ou de celui de la simple lecture dilettante.

En amont, la posture de l'auteur en regard de ses récits est triple. AngeÉmile Maheu endosse successivement plusieurs rôles. Il se fait tour à tour ethnographe, narrateur et auteur. Au cours de sa longue carrière de conteur et d'enseignant, il a archivé dans sa mémoire quantité de contes d'origine diverse - cela est visible dans l'énumération succincte qu'il dresse de ses sources dans le conte «Ti-Jean et la Vallée des âges » (p. 265) - de légendes, de croyances populaires et même d'un fait divers. Curieusement, cette historiette, pour reprendre une expression chère à $\mathrm{M}^{\mathrm{me}}$ de La Fayette, ne figure pas parmi les 45 titres recensés dans la table des matières. On la retrouve uniquement sur DC en cinquième position : «Le Docteur, le radium et le biscuit». À l'audition, le biscuit en question s'avère dans les faits un « beigne ». Ce récit n'est pas sans rappeler ces textes que publiait naguère Sélection du Reader's Digest : « L'être le plus extraordinaire que j'ai connu ».

L'ensemble des textes provient de l'enfance beauceronne de Maheu d'où le clin d'œil complice à Robert Cliche dans « La Réparation d'honneur » (p. 203) - de ses contacts avec les milieux traditionnels de transmission (camp de bûcherons dans les chantiers du Maine) et de ses lectures qu'on devine nombreuses : le Méphintos (p. 232) dans «Ti-Jean et les trois colombes » (p. 225) n'est pas sans évoquer le Méphistophélès du Faust de Gœthe. De l'aveu même de l'auteur anonyme de la « Présentation », bien qu'il puisse 
s'agir de Maheu lui-même, le conteur s'est mis sous la dictée de sa mémoire pour consigner par écrit ce qu'elle avait archivé, devenant par cela l'ethnographe de lui-même doublé pour la circonstance d'un compilateur. Cette activité recouvre les deux autres rôles. Il s'est fait le porteur de la tradition orale lors de ses performances publiques à un double titre encore une fois : comme conteur en narrant des contes bien typés et comme narrateur en relatant des légendes, des croyances populaires, allant même jusqu'à endosser le rôle de témoin oculaire dans la relation d'une légende bien connue : « La Chasse-galerie au Camp à Blanchette » (p. 68). Il serait étonnant dans ce cas que Maheu ait pu participer de quelque manière que ce soit à cette « chasse volante ». La dernière phrase du récit en laissera plus d'un dubitatif.

Enfin l'auteur a franchi la ligne tracée en pointillé qui sépare la narration de la création. Trois textes nommément désignés, relèvent de cette catégorie. En note liminaire à « Tom-Pouce au palais de la Fée des roses » (p. 11), l'auteur avoue sans ambages : «[...] j'ai créé dix aventures de Tom-Pouce ». Celle qui nous est présentée n'en demeure pas moins fermement lestée par la tradition orale. « La Boule bleue» (p. 32) nous offre un récit d'anticipation qui se développe malgré tout à l'intérieur des normes du conte traditionnel. En fait, les deux héros qui évoluent dans un passé récent font un bond dans un futur proche pour ne découvrir en somme que ce qui existe maintenant : «Dans quelques années, ces écrans-animés [la télévision] seront très populaires sur la Terre» (p. 41). Le cadre, bien que mâtiné de merveilleux scientifique, reprend des personnages propres aux Mille et une Nuits. Aussi ne faut-il pas s'étonner de rencontrer un «Vizir» et un «Émir Royal» (p. 42). La dernière création, « La Poubelle parlante » (p. 189), est un emprunt fait à un élève de l'école secondaire Sainte-Marie de New-Liskeard, Steve Arnold.

Le sous-titre du recueil - Contes traditionnels - peut, en ce sens, induire en erreur un lecteur non averti, car les genres majeurs de l'orature - le conte, la légende, le mythe - sont généralement source de confusion autant chez les praticiens que chez les amateurs de tels récits. Ceux qui œuvrent dans le milieu de l'enseignement ne sont pas exempts non plus de cette ambiguité et l'absence d'une classification minimale n'arrangera pas les choses, au contraire. On devine aisément qu'on y puisera des récits qu'on prendra indifféremment pour des contes ou des légendes, les deux risquant de s'équivaloir dans l'esprit de nombreux enseignants. Les débats récents soulevés par la parution du Da Vinci Code de Dan Brown prouvent, si besoin en est, que la confusion des genres traverse tous les milieux intellectuels. À cet effet, l'ouvrage d'Ange-Émile Maheu pourrait donner lieu à un bel exercice scolaire : suivant quels critères peut-on démêler et attribuer à leur genre particulier les récits proposés ? En somme, il y a tout un travail d'identification 
et de classification à effectuer selon la dichotomie éprouvée : nommer/classer. Car, il faut bien se rendre à l'évidence, Mon oncle Émile conte compile des récits dans un joyeux désordre qui en fait le degré zéro de la classification. La seule justification de leur présence et de leur agencement se trouve dans le goût personnel et légitime de l'oncle en question, et l'unité de l'ensemble repose sur le style du conteur dont la signature ne se dément pas d'un texte à l'autre.

Cela étant, on peut légitimement supposer que le public auquel s'adresse Maheu n'est pas celui des spécialistes de la chose. Il « surfe » sur la déferlante du renouveau du conte qui table sur l'intérêt indéniable d'un public qui, toutes catégories confondues, aspire au réenchantement du monde et à la convivialité retrouvée grâce à la présence physique et chaleureuse du conteur. Le style narratif de l'auteur épouse cette considération : ni transcription fidèle d'une narration antécédente ni écriture travaillée au sens étroit du terme. Parlons plutôt d'une écriture oralisante où les oreilles trouvent davantage leur plaisir que les yeux parce que le lecteur a l'impression d'entendre ce qu'il lit proféré par la bouche même du conteur.

Le recueil a été écrit dans un style familier, direct, concis. L'auteur n'a que faire des images savoureuses dont se délectent des lecteurs gourmands. Il ne quitte pas ses héros des yeux, s'attachant à leurs pas : les motifs se succèdent les uns aux autres sans qu'une image fournisse l'excuse commode d'une pause qui permettrait d'approfondir tel épisode tout en s'en imprégnant. Le récit inauguré, la trame narrative chemine inéluctablement vers la fin.

Le disque compact qui accompagne le livre permet à l'auditeur de se familiariser avec le style oral du conteur. La diction est claire sans être recherchée, la langue familière et en général correcte. Ange-Émile Maheu est un narrateur chaleureux sans être racoleur. Ceux qui voudraient accroître leur plaisir en suivant le conteur texte en main en seront pour leur peine. Si l'auteur a compilé les divers récits sous la dictée de sa mémoire, cette mémoire était plus littéraire qu'orale, ce qui explique le fossé séparant ce qu'on lit de ce qu'on entend. Mais cela ne doit pas nous décevoir ni nous dérouter. Il faut prendre chaque procédé narratif dans ce qu'il a d'irréductible à l'autre et convenir une fois pour toutes que tout individu est essentiellement bilingue à l'intérieur de sa langue maternelle. La langue parlée, héritée de ses parents et relayée plus tard par le milieu, obéit à des normes qui ne rencontrent pas nécessairement celles de la langue écrite. Ce que nous dicte notre mémoire dans l'intimité de la narration mentale n'adopte pas le même véhicule pour accomplir le même parcours selon que la voie empruntée passe par l'écriture ou par l'orature. Maheu nous en administre empiriquement la sage leçon.

Il est dommage pour la qualité générale de l'ouvrage que l'éditeur n'ait pas porté plus d'attention à certains détails qui agacent tout en demeurant 
mineurs. Ainsi, il aurait dû mettre la table des matières du disque compact sur le rabat de la quatrième de couverture pour en faciliter la consultation au lieu de se contenter de l'imprimer uniquement sur le disque lui-même. De plus, une lecture supplémentaire par le correcteur d'épreuves aurait permis à celui-ci d'éliminer les dernières coquilles du texte et quelques incohérences vénielles. « Ça été » ( p. 73, 211 et 271) aurait retrouvé son verbe (ç’a été) et « gentlemen» (p. 21) son nombre (gentleman). L'adverbe « icitte» qui saupoudre tout le texte a été imprimé en caractères réguliers en dépit du fait qu'il figure au glossaire. «Moé le Roé » (p. 167) n'aurait pas rougi de se voir drapé de l'italique et l'expression « oreille de crisse » (p. 240) méritait une mention d'honneur à ce même glossaire. On s'étonnera de ne pas rencontrer Les Contes de Jos Violon de Louis Fréchette dans la bibliographie. Pourtant, la description des lutins dans la légende éponyme (p. 104) s'en inspire beaucoup.

Mais ce sont là des contrariétés qu'il serait injuste de retenir contre l'ensemble du recueil qui propose une agréable incursion à travers la tradition orale bien que les lieux visités se révèlent sans surprises. Encore là, cette dernière particularité est garante de la durée et tributaire de la mémoire collective. L'inédit ne l'est vraiment que lors de sa première occurrence. Après, il fait partie des formes reçues, mais s'il n'est pas repris, il risque de disparaître. En dernier ressort, ne reste vraiment que le style qui, selon l'heureuse formule de Buffon " est l'homme même ». C'est lui qui attire de prime abord, qui retient ensuite et qui pousse à en redemander encore et encore. À cet égard, Ange-Émile Maheu sait retenir notre attention.

\section{BERTRANd Bergeron}

Collège d'Alma

NoËL, FrançoIse. Family Life and Sociability in Upper and Lower Canada, 1780-1870. A View from Diaries and Family Correspondance. Montréal \& Kingston, McGill-Queen's University Press, 2003, 372 p. ISBN 0773524452.

C'est par le biais de la littérature personnelle que l'historienne Françoise Noël porte un regard sur la vie familiale et sociale aux Haut et Bas-Canada entre les années 1780 et 1870 . À cet effet, l'auteur a consulté une douzaine de journaux intimes et plus d'une trentaine de fonds de papiers de famille, conservés aux Archives de l'Ontario et aux Archives nationales du Canada. Les diaristes et les épistoliers choisis, il fallait s'y attendre, appartiennent au 